\title{
THE CONJUGATING REPRESENTATION \\ OF A SEMISIMPLE ALGEBRAIC GROUP
}

BY R. W. RICHARDSON

\author{
Communicated by Hyman Bass, July 12, 1976
}

Let $G$ be a connected, simply connected semisimple algebraic group over an algebraically closed field $k$ of characteristic zero and let $A(G)$ denote the algebra of regular functions on $G$. We let $G$ act morphically on $G$ by conjugation. This defines a representation of $G$ on $A(G)$. In analogy with the terminology for finite groups we call this the conjugating representation of $G$. Let $C(G)$ be the algebra of all (regular) class functions on $G$; equivalently $C(G)=A(G)^{G}$. Let $\Lambda$ be a set of representatives for the equivalence classes of irreducible rational representations of $G$. If $\lambda: G \rightarrow \operatorname{GL}\left(V_{\lambda}\right)$ is an element of $\Lambda$, let $d_{\lambda}=\operatorname{dim} V_{\lambda}$ and let $m_{\lambda}=$ $\operatorname{dim} V_{\lambda}^{T}$, where $T$ is a maximal torus of $G$. Let $A(G)_{\lambda}$ denote the isotopic component of $A(G)$ of type $\lambda$. Each $A(G)_{\lambda}$ is a finitely generated $C(G)$-module and $A(G)$ is the $C(G)$-module direct sum of the $A(G)_{\lambda}, \lambda \in \Lambda$.

THEOREM A. Let $\lambda \in \Lambda$. Then there exists a G-stable vector subspace $H_{\lambda}$ of $A(G)_{\lambda}$ such that the $k$-linear map $C(G) \otimes_{k} H_{\lambda} \rightarrow A(G)$ defined by $c \otimes h \rightarrow$ ch is an isomorphism of vector spaces. The rational $G$-module $H_{\lambda}$ is equivalent to the direct sum of $m_{\lambda}$ copies of $V_{\lambda}$. In particular, $A(G)_{\lambda}$ is a free $C(G)$-module of rank $d_{\lambda} m_{\lambda}$ and $A(G)$ is a free $C(G)$-module.

For the adjoint action of $G$ on the Lie algebra $g$ and the corresponding representation of $G$ on the algebra $A(g)$ of polynomial functions on $g$, a similar theorem was proved by Kostant [1]. In the Lie algebra case, the proof of freeness is greatly simplified by the graded algebra structure of $A(\mathrm{~g})$. Our proof makes use of the results of Steinberg [4] (see also [3], [5]) on conjugacy classes in $G$ and on the fibres of the morphism $\pi: G \rightarrow k^{r}, r=\operatorname{rank} G$, given by the fundamental characters. We use the methods of commutative algebra. In particular, we require two separate applications of the Serre conjecture on projective modules over polynomial rings, which has recently been proved by Quillen [2] .

If the base field $k$ is of prime characteristic, we have a different proof that $A(G)$ is a free $C(G)$-module. In this case we have not been able to prove the existence of a $G$-stable vector subspace $H$ of $A(G)$ such that the product map $C(G) \otimes_{k} H \rightarrow A(G)$ is a vector space isomorphism.

In $\S 1$ we outline the proof of Theorem A. A detailed proof will appear elsewhere.

AMS (MOS) subject classifications (1970). Primary 20G05; Secondary $18 \mathrm{~F} 25$. 
1. Sketch of the proof of Theorem A. Roughly, there are three steps in the proof: (1) prove that $A(G)_{\lambda}$ is a projective $C(G)$-module; (2) apply the Serre conjecture to prove that $A(G)_{\lambda}$ is a free $C(G)$-module (Steinberg has shown that $\left.C(G) \cong k\left[X_{1}, \ldots, X_{r}\right]\right)$; (3) apply the Serre conjecture again to find a $G$-stable subspace $H_{\lambda}$ as in Theorem A.

STEP (1). The projectivity of $A(G)_{\lambda}$ follows from Steinberg's results and 1.1 and 1.2 below.

1.1. Let $C$ be a Noetherian integral domain which is a Jacobson ring and let $M$ be a finitely generated $C$-module. Assume that there exists an integer $q$ such that, for every maximal ideal $m$ of $C$, the dimension of the $(C / m)$-vector space $M / m M$ is equal to $q$. Then $M$ is a projective $C$-module of rank $q$.

1.2. Let $x$ be a regular element of $G$. Then, for every $\lambda \in \Lambda, \operatorname{dim} V_{\lambda}^{T}=$ $\operatorname{dim} V_{\lambda}^{Z}{ }^{(x)}$.

STEP (2). The result below is due to Quillen [2]. The problem was posed almost twenty years ago by Serre and has become known as the Serre conjecture.

2.1. Let $L$ be a principal ideal domain and let $M$ be a finitely generated projective module over the polynomial ring $L\left[X_{1}, \ldots, X_{r}\right]$. Then $M$ is a free module.

Since $C(G)$ is a polynomial ring over $k$ and $A(G)_{\lambda}$ is a projective $C(G)$ module, it follows that $A(G)_{\lambda}$ is free.

STEP (3). Let $\left(f_{1}, \ldots, f_{n}\right)$ be a basis of the free $C(G)$-module $A(G)_{\lambda}$; here $n=d_{\lambda} m_{\lambda}$. The vector subspace of $A(G)_{\lambda}$ spanned by $f_{1}, \ldots, f_{n}$ is not necessarily $G$-stable. We wish to find a basis which spans a $G$-stable subspace. Let $g \in G$. Then $g \cdot f_{j}=\Sigma_{i=1}^{n} \alpha_{i j}(g) f_{i}$, where each $\alpha_{i j}(g)$ is an element of $C(G)$. This determines a homomorphism (of abstract groups) $\alpha: G \longrightarrow$ $\mathrm{GL}_{n}(C(G)), \alpha(g)=\left(\alpha_{i j}(g)\right)$. Identify $C(G)$ with the algebra of polynomial functions on $k^{r}$ and define $\eta: G \times k^{r} \rightarrow \mathrm{GL}_{n}(k)$ by $\eta(g, y)=\left(\alpha_{i j}(g)(y)\right)$. With some work we can prove that $\eta$ is a morphism of algebraic varieties. For each $y \in k^{r}$, define a rational representation $\eta_{y}: G \rightarrow \mathrm{GL}_{n}(k)$ by $\eta_{y}(g)=\eta(g, y)$. Then $\left(\eta_{y}\right)_{y \in k^{r}}$ is a parametrized family of equivalent representations of $G$ on $k^{n}$. Let $R$ be the set of all rational representations of $G$ on $k^{n}$ which are equivalent to $\eta_{0}$. Then $\mathrm{GL}_{n}(k)$ acts transitively on $R$ and the stabilizer, $H$, of $\eta_{0}$ is isomorphic to $\mathrm{GL}_{m_{\lambda}}(k)$. Thus $\eta$ determines a map $\beta: k^{r} \rightarrow \mathrm{GL}_{n}(k) / H$ and this map is a morphism. (The easiest way to see this is to consider the corresponding Lie algebra representations $d \eta_{y}: g \rightarrow g L_{n}(k)$.) Now $\mathrm{GL}_{n}(k) \rightarrow \mathrm{GL}_{n}(k) / H$ is a locally trivial principal $H$-bundle and $H$ is isomorphic to $\mathrm{GL}_{m_{\lambda}}(k)$. Hence $\beta$ determines a vector bundle of rank $m_{\lambda}$ on $k^{r}$. Since vector bundles on $k^{r}$ correspond to projective $C(G)$-modules, the Serre conjecture implies that this vector bundle is trivial. The triviality of this vector bundle allows us to find a new basis of the $C(g)$-module $A(G)_{\lambda}$ such that the vector subspace $H_{\lambda}$ spanned by this basis is $G$-stable. This completes the outline of the proof. 


\section{REFERENCES}

1. B. Kostant, Lie group representations on polynomial rings, Amer. J. Math. 85 (1963), 327-404. MR 28 \#1252.

2. D. Quillen, Projective modules over polynomial rings (to appear).

3. T. A. Springer and R. Steinberg, Conjugacy classes, Seminar on Algebraic Groups and Related Finite Groups (Inst. for Adv. Study, Princeton, N. J., 1968/69), Lecture Notes in Math., vol. 131, S pringer, Berlin, 1970, pp. 167-266. MR $42 \# 3091$.

4. R. Steinberg, Regular elements of semisimple algebraic groups, Inst. Hautes Études Sci. Publ. Math. No. 25 (1965), 49-80. MR 31 \#488.

5. - Conjugacy classes in algebraic groups, Lecture Notes in Math., vol. 366, Springer-Verlag, Berlin and New York, 1974. MR 50 \#466.

DEPARTMENT OF MATHEMATICS, UNIVERSITY OF DURHAM, DURHAM CITY, UNITED KINGDOM 